\title{
Análise dos efeitos da auriculoterapia e ventosaterapia no desempenho laboral de colaboradores de uma instituição de ensino privada em Teresina- PI
}

Analysis of the effects of auriculotherapy and wind therapy on the work performance of employees of a private educational institution in Teresina - PI

Análisis de los efectos de la auriculoterapia y la eólica en el desempeño laboral de los empleados de una institución educativa privada en Teresina - PI

Sara Ferreira Lobato de Brito ORCID: https://orcid.org/0000-0001-8207-7906 Centro Universitário Unifacid, Brasil E-mail: Saraflbrito99@gmail.com Amanda Sérvio Salazar ORCID: https://orcid.org/0000-0002-3222-7803 Centro Universitário Unifacid, Brasil E-mail: amandaservios@gmail.com

Maria Eugênia Macedo Teixeira ORCID: https://orcid.org/0000-0002-6754-4446 Centro Universitário Unifacid, Brasil

E-mail: mariaeugenia.teixeira@ hotmail.com

Victoria Caroline Oliveira Pinto ORCID: https://orcid.org/0000-0002-6143-7068 Centro Universitário Unifacid, Brasil E-mail: viccarolineo252@gmail.com

Eulália Luana Rodrigues da Silva ORCID: https://orcid.org/0000-0002-9605-2037

Centro Universitário Unifacid, Brasil

E-mail: eulalialuana011@hotmail.com

Valéria Alves da Rocha

ORCID: https://orcid.org/0000-0003-4667-5153 Centro Universitário Unifacid, Brasil E-mail: Valeriarocha_10@hotmail.com

Marcélia Gomes Silva

ORCID: https://orcid.org/0000-0002-5081-6388

Centro Universitário Unifacid, Brasil

E-mail: marceliasilva118@gmail.com

Bianca Gisele Lira Gonzaga

ORCID: https://orcid.org/0000-0002-0615-8242

Centro Universitário Unifacid, Brasil

E-mail: biancagonzagapi@gmail.com

Francisco Tassio Azevedo Teixeira ORCID: https://orcid.org/0000-0002-8994-8249 Centro Universitário Unifacid, Brasil E-mail: tassioazevedo@hotmail.com

Marcello Alencar Silva

ORCID: https://orcid.org/0000-0001-9451-2979 Centro Universitário Unifacid, Brasil

E-mail: ft.alencar@gmail.com

Maura Cristina Porto Feitosa

ORCID: https://orcid.org/0000-0001-7095-7228 Universidade Estadual do Piauí, Brasil E-mail: mauraportofisio@ hotmail.com

Veruska Cronemberger Nogueira Rêbelo ORCID: https://orcid.org/0000-0002-5013-4432 Universidade Estadual do Piauí, Brasil E-mail: veruskanogueirarebelo@yahoo.com.br Neusa Barros Dantas Neta ORCID: https://orcid.org/0000-0001-7127-1463 Centro Universitário Unifacid, Brasil E-mail: nbdn2@msn.com 


\title{
Resumo
}

Introdução: A Medicina Tradicional Chinesa (MTC), dispõe de várias técnicas, dentre elas a ventosaterapia e auriculoterapia, viáveis pelo baixo custo e seus efeitos, melhora álgica, do sono e relaxamento. Objetivo: Avaliar por meio das respectivas técnicas, os benefícios sobre as Doenças Ocupacionais Relacionadas ao Trabalho (DORTs), produtividade laboral e qualidade de vida dos colaboradores de uma instituição de ensino privado. Metodologia: Trata-se de uma pesquisa de campo, do tipo descritivo, prospectivo, experimental com abordagem qualiquantitativa, a amostra foi composta por 20 colaboradores. Para a coleta de dados utilizou-se uma ficha de avaliação elaborada pelo próprio pesquisador relacionada a atividade laboral, a escala visual analógica (EVA) e o Inventário Breve de Dor (BPI). Ao final da coleta os dados estes foram tabulados em planilhas eletrônicas, por meio do programa Microsoft Excel 2016 e exportados para o software SPSS 23.0. Resultados: Houve prevalência do gênero feminino, 55\% ( $\mathrm{n}=11$ ), destes $25 \%(\mathrm{n}=5)$ dos participantes desempenham a função de assistente acadêmico. Em relação a queixa principal, $35 \%(n=7)$ queixaram-se de tensão muscular no trapézio, e a ventosaterapia foi a intervenção que prevaleceu $(60 \%)$. A média de dor variou no início entre 4 e 7 , após o procedimento diminuiu para $2( \pm 0,89)$, já na qualidade de vida, o item "trabalho" teve melhor resultado, variando a média de 5,40 $(2,76)$ para 0,00 $(0,00)$. Conclusão: A aplicação da ventosaterapia e auriculoterapia como intervenção proporcionaram melhora dos sintomas das DORT's, da produtividade laboral e qualidade de vida dos trabalhadores.

Palavras-chave: Medicina tradicional chinesa; Qualidade de vida; Desempenho laboral.

\begin{abstract}
Introduction: Traditional Chinese Medicine (TCM) has several techniques, including wind therapy and auriculotherapy, which are viable due to their low cost and their effects, improving sleep and relaxation. Objective: to evaluate, through the respective techniques, the benefits on Work-related Occupational Diseases (WCDs), labor productivity and quality of life of the employees of a private educational institution. Methodology: This is a field research, descriptive, prospective, experimental with a qualiquantitative approach, the sample was composed of 20 collaborators. For data collection we used an evaluation form developed by the researcher himself related to labor activity, the visual analog scale (EVA) and the Brief Inventory of Pain (BPI). At the end of the collection the data were tabulated in spreadsheets, using the Microsoft Excel 2016 program and exported to the SPSS 23.0 software. Results: There was a prevalence of female gender, $55 \%(n=11)$, of these $25 \%(n=5)$ of participants perform the function of academic assistant. Regarding the main complaint, 35\% ( $\mathrm{n}=7)$ complained of trapezium muscle tension, and wind therapy was the prevailing intervention (60\%). The mean pain varied at the beginning between 4 and 7 , after the procedure it decreased to $2( \pm 0.89)$, already in the quality of life, the item "work" had better result, varying the mean from $5.40(2.76)$ to $0.00(0.00)$. Conclusion: The application of wind therapy and auriculotherapy as an intervention provided improvement in the symptoms of MSDs, labor productivity and quality of life of workers.
\end{abstract}

Keywords: Traditional chinese medicine; Quality of life; Work performance.

\section{Resumen}

Introducción: La Medicina Tradicional China (MTC) tiene varias técnicas, entre ellas la terapia de succión y la auriculoterapia, que son viables por su bajo costo y efectos, mejora el dolor, el sueño y la relajación. Objetivo: evaluar, a través de las técnicas respectivas, los beneficios en Enfermedades Laborales Relacionadas con el Trabajo (EMSP), la productividad laboral y la calidad de vida de los empleados de una institución educativa privada. Metodología: Se trata de una investigación de campo, descriptiva, prospectiva, experimental, con enfoque cualitativo y cuantitativo, la muestra estuvo conformada por 20 colaboradores. Para la recolección de datos se utilizó un formulario de evaluación elaborado por el investigador relacionado con la actividad laboral, la escala visual analógica (EVA) y el Inventario Breve de Dolor (BPI). Al final de la recopilación, los datos se tabularon en hojas de cálculo electrónicas utilizando el programa Microsoft Excel 2016 y se exportaron al software SPSS 23.0. Resultados: Hubo una prevalencia de mujeres, 55\% $(\mathrm{n}=11)$, de las cuales el 25\% $(\mathrm{n}=5)$ de los participantes realiza el rol de asistente académico. En cuanto a la queja principal, el 35\% $(\mathrm{n}=7)$ se quejó de tensión muscular en el trapecio y la terapia de succión fue la intervención más prevalente $(60 \%)$. El dolor promedio varió de 4 a 7 , luego del procedimiento disminuyó a $2( \pm 0.89)$, y en términos de calidad de vida, el ítem "trabajo" tuvo mejores resultados, con una media de $5.40(2,76)$ a $0,00(0,00)$. Conclusión: La aplicación de la terapia de succión y auriculoterapia como intervención proporcionó una mejoría en los síntomas de WMSD, la productividad laboral y la calidad de vida de los trabajadores. Palabras clave: Medicina tradicional china; Calidad de vida; Desempeño laboral. 


\section{Introdução}

$\mathrm{O}$ trabalho, enquanto fator determinante do desenvolvimento humano, tem representado um papel de grande importância na história da humanidade. A salubridade do ambiente de trabalho é, sem dúvida, um fator relevante em matéria de saúde e bem-estar das populações, uma vez que trabalhadores saudáveis e seguros em locais de trabalho saudáveis e seguros são, por certo, mais produtivos e, dessa forma, contribuintes para o desenvolvimento econômico que o trabalho pode proporcionar às sociedades modernas (UVA, 2009).

De acordo com os dados da Organização Internacional do Trabalho (OIT) ao analisar os acidentes e doenças ocupacionais divulgou que $14 \%$ dos benefícios anuais de saúde foram relacionados a desordens mentais. É notório que o mundo do trabalho atual, dados os desafios do progresso industrial, globalização, desenvolvimento tecnológico e comunicação virtual, impõe condições que excedem os limites das habilidades e competências das pessoas, que gera como resultado o estresse no ambiente de trabalho, podendo causar disfunções físicas, psicológicas e sociais que interferem nas condições de saúde dos trabalhadores e minam a produtividade (OIT, 2016).

$\mathrm{O}$ aumento da carga de trabalho, longos expedientes e o estresse laboral faz com que o trabalhador tenha maior predisposição as lesões por esforços repetitivos (LER) e distúrbios osteomusculares relacionado ao trabalho (DORT) as quais estão atribuídas as principais causas das doenças laborais. Segundo Alencar e Merlo (2018) as doenças ocupacionais relacionadas ao trabalho são processos patológicos de etiologia multifatorial que levam a incapacidade do trabalhador, trazendo a prejuízos tanto para o empregador quando para o empregado. Para que se estabeleça uma relação entre o trabalho e o surgimento de uma doença é importante que se compreenda que saúde é um "completo estado de bem-estar físico, social e metal e não apenas a ausência de doença". Desse modo um desequilíbrio em uma dessas áreas causará no indivíduo uma injúria ou processo patológico (WHO, 2006).

Segundo o Ministério da Saúde (2008), cuidar significa oferecer ao outro, em forma de serviço, o cuidado; perceber a outra pessoa como ela é, e como se mostra, seus gestos e falas, sua dor e limitação. Com base nisso, a Fisioterapia como ciência que avalia, diagnostica e trata as disfunções que promovem dores, limitações e perda da qualidade de vida, destaca-se pelo manuseio das técnicas da Medicina Tradicional Chinesa (MTC) promove a integração e interação entre o ser humano e a natureza, a manutenção da saúde e a prevenção da doença, visando a harmonizar o estado de saúde geral das pessoas (Matos \& Pires, 2010). Com base na MTC, o estado de saúde corresponde a um estado de equilíbrio, enquanto as doenças são vistas como uma ruptura, podendo ser condicionada pelo estresse, desencadeando uma má distribuição energética nos meridianos (canais por onde circula o Qi ou energia), órgãos e vísceras (Nascimento, 2006).

Partindo disso, diversos estudos têm descrito a MTC como uma forma não farmacológica para tratar distúrbios osteomusculares e viscerais por meio da aplicação de um conjunto de técnicas orientais: acupuntura, moxabustão, acupressão, fitoterapia, exercícios físicos, auriculoterapia, reflexologia dos pés, craniopuntura entre outros (Lu et al., 2004).

Dentre estas, destaca-se o uso da ventosaterapia e auriculoterapia como uma das mais utilizadas em vista do baixo custo, segurança e eficácia. A ventosaterapia consiste na aplicação de copos de vidro ou acrílico que por meio de uma pressão negativa criam um vácuo, o que gera uma sucção da pele e musculatura para dentro do recipiente, estimulando a circulação sanguínea e com este processo são liberadas as toxinas existentes no sangue, melhorando assim o fluxo energético (Kim, 2002).

Enquanto a auriculoterapia, é uma técnica de diagnóstico e tratamento realizada por meio de estímulos no pavilhão da orelha, onde a orelha simboliza um feto de cabeça para baixo, sendo considerada assim uma área reflexa. O tratamento se dá pela colocação de sementes de mostarda, esferas de ouro ou prata e agulhas semipermanentes nas áreas reflexas da orelha, sendo assim, o tipo de material será escolhido de acordo com a sintomatologia do paciente (UVA, 2005). 
Diante da eficácia das técnicas citadas e a intensa desordem causada pela sobrecarga de trabalho apresentada nos colaboradores do ensino superior, o objetivo do estudo foi investigar os efeitos da auriculoterapia e ventosaterapia sob o desempenho laboral dos colaboradores de um Centro Universitário de Teresina-PI.

\section{Metodologia}

\subsection{Princípios éticos}

A presente pesquisa foi realizada de acordo com as diretrizes para pesquisa envolvendo humanos preconizadas pela Resolução do Conselho Nacional de Saúde 466/12, sendo a mesma submetida à apreciação do Comitê de Ética em Pesquisa com Seres Humanos do Centro Universitário UNIFACID e aprovada sem restrições sob o número de parecer 3.617.191 e CAAE 21768619.2.0000.5211.

\subsection{Desenho e participantes}

Trata-se de uma pesquisa de campo, experimental, do tipo descritivo, com abordagem qualitativa e quantitativa, realizada em uma Centro Universitário, localizado em Teresina - PI, entre o período de agosto de 2019 a março de 2020. A população acessível deste estudo foi composta dos profissionais atuantes na referida instituição.

Foram incluídos colaboradores com vínculo empregatício, com carga horária entre 30 a 40 horas semanais, sem distinção de gênero e idade, que apresentaram queixas físicas, que estivessem refletindo na sua atividade laboral e/ou qualidade de vida, e que tivessem disponibilidade de tempo para a realização da terapia, mantendo um mínimo de atendimento estipulado para a obtenção de resultados significativos. Foram excluídos da pesquisa os colaboradores que apresentavam dor secundária de uma lesão ortopédica e/ou traumática, que faziam uso de algum fármaco analgésico e/ou anti-inflamatório e que estivesse sob atendimento fisioterapêutico.

\subsection{Instrumentos de Avaliação}

Os dados foram coletados por meio de uma entrevista semiestruturada, dividida em três momentos: No primeiro, foi caracterizado o perfil profissional dos colaboradores, compreendendo idade, gênero e o cargo de ocupação. No segundo momento, foi investigado o perfil da dor quanto a sua localização, intensidade e o impacto sobre seu desempenho laboral. No terceiro momento, foi realizado uma análise comparativa entre as técnicas aplicadas, seguido da reavaliação da dor.

Para a avaliação da intensidade da dor foi utilizado a Escala visual analógica (EVA): A escala trata-se de uma linha com as extremidades numeradas de 0-10. Em uma extremidade da linha é marcada "nenhuma dor" e na outra "pior dor inimaginável". Pede-se, então, para que o paciente avalie e marque uma linha na dor presente naquele momento (Martinez, Grassi \& Marques, 2011).

O Inventário Breve de Dor foi aplicado visando analisar a intensidade, interferência da dor na habilidade para caminhar, atividades diárias do paciente, no trabalho, atividades sociais, humor e sono. Trata-se de um instrumento multidimensional, que faz uso de uma escala de 0-10. A dor avaliada pelo paciente é aquela presenciada no momento do questionário e a mais intensa, a menos intensa e a média da dor das últimas 24 horas.

Todas as escalas de avaliação aplicadas antes da primeira intervenção a cada 30 dias, durante 7 meses. Cada paciente foi atendido 1 vez na semana, por 30 minutos gerando 4 atendimentos durante um mês, totalizando 28 atendimentos ao final da coleta de dados. 


\subsection{Análise Estatística}

Os dados foram tabulados em planilhas eletrônicas, por meio do programa Microsolft Excerl 2016 e exportados para o software SPSS 23.0. Foram verificadas as pressuposições de normalidade dos dados pelo teste Shapiro-Wilk ao nível de significância de $5 \%$ e as significâncias das médias foram validadas pelo teste $\mathrm{t}$ para amostras pareadas com intervalo de confiança de $95 \%$ e significância $\mathrm{p}<0,05$.

\section{Resultados e Discussão}

No presente estudo foram incluídos 20 participantes, com maior predomínio para o gênero feminino $55 \%$ (n=11), com idade média de 33 anos $( \pm 7,15)$, sendo a maioria dos colaboradores desempenham a função de assistente acadêmicos $(25 \%$; $\mathrm{n}=5$ ), como mostra a Tabela 1 .

Tabela 1. Caracterização profissional dos colaboradores do Centro Universitário. Teresina, PI ( $n=20)$.

\begin{tabular}{|c|c|c|c|}
\hline & DPM & $\mathbf{n}$ & $\%$ \\
\hline Idade & $33,30( \pm 7,15)$ & & \\
\hline 22 a 28 & & 7 & 35,0 \\
\hline 29 a 34 & & 3 & 15,0 \\
\hline 55 a 40 & & 7 & 35,0 \\
\hline 41 a 47 & & 3 & 15,0 \\
\hline \multicolumn{4}{|l|}{ Gênero } \\
\hline Masculino & & 9 & 45,0 \\
\hline Feminino & & 11 & 55,0 \\
\hline \multicolumn{4}{|l|}{ Profissão/Ocupação } \\
\hline Marketing & & 2 & 10,0 \\
\hline Auxiliar de manutenção & & 1 & 5,0 \\
\hline Assistente Acadêmico & & 5 & 25,0 \\
\hline Analista Acadêmico & & 1 & 5,0 \\
\hline Assistente de Operações & & 1 & 5,0 \\
\hline Consultor & & 3 & 15,0 \\
\hline Porteiro & & 2 & 10,0 \\
\hline Técnico de Laboratório & & 2 & 10,0 \\
\hline Supervisor de Almoxarifado & & 1 & 5,0 \\
\hline Supervisor de Captação & & 1 & 5,0 \\
\hline Professor & & 1 & 5,0 \\
\hline
\end{tabular}

Legenda: Desvio Padrão da Média (DPM); Número (n); Percentual (\%); Fonte: Autores (2020).

Semelhante ao resultado apresentado quanto ao predomínio de gênero, Pandolphi e Costa (2016) ao analisarem a amostragem de seus dados, observaram que houve predomínio para o gênero feminino. Os autores justificam que as mulheres apresentam maior acometimento por DORT em vista das diferenças anatomofisiológicas e hormonais.

De acordo com Silva (2016), o uso de salto alto por mais de quatro horas diárias pode promover alterações de equilíbrio em mulheres jovens, provavelmente devido ao desarranjo muscular/encurtamento de cadeia posterior, o que leva às alterações posturais e do centro de gravidade. Além disso, mulheres são expostas a um regime de trabalho duplo, compreendendo a vida familiar, tais como cuidar da casa e filho, e no próprio local de trabalho, levando a sobrecarga física e mental, que posteriormente resulta no processo de adoecimento. Dado semelhante foi encontrado no estudo de Rodrigues et al (2021) ao analisar o impacto do uso de calçados com salto de colaboradoras de uma instituição de ensino superior, os autores descrevem as principais complicações apresentadas, dentre elas o quadro álgico e a perda da qualidade de vida, o que implica diretamente na postura e desempenho do colaborador. 
Semelhante aos dados obtidos neste estudo, ao qual as mulheres foram as mais afetadas pelas atividades laborais, Moreno, et al. (2021) descrevem que um dos fatores determinantes o gênero feminino seja o mais afetado está relacionado a fisiologia hormonal, uma vez que o estrógeno, hormônio predominante, acentuam os processos degenerativos articulares, afetando as estruturas ósseas e cartilagem articular, gerando uma resposta inflamatória, o que causa um desarranjo global, o que justificaria os dados apresentados.

Outro achado importante é que 25\% (n=5) dos voluntários, desempenham a função de assistente acadêmicos, função ao qual o colaborador é responsável pelo atendimento ao público para resolução financeira do setor estudantil, estes são expostos a uma longa carga horária de trabalho frente ao computador, o que leva a quadros de estresse, tensão psicológica e dores crônicas geradas pela necessidade de realização de múltiplas tarefas simultaneamente. Concomitante ao resultado apresentado, Alencar (2018) discorreu em seu trabalho que o expediente extenuante se torna cansativo e predispõe ao servidor ao desenvolvimento de LER e DORT. Em contrapartida, Viegas e Almeida (2016) acreditam que as manifestações dolorosas dentro do ambiente de trabalho são ocasionadas pelo próprio indivíduo e que estão relacionadas aos fatores como: posturas inadequadas, movimentos repetitivos e a permanência por longos períodos em uma mesma posição.

A Tabela 2 descreve a queixa principal relatada pelos colaboradores e a intervenção realizada nos mesmos como modo de amenizar o quadro álgico. $35 \%(n=7)$ dos avaliados queixam-se de tensão muscular no trapézio e 25\% (n=5) relata ter dor lombar crônica (Tabela 2). Quanto a técnica aplicada, a ventosaterapia se destacou sendo a mais aplicada tanto de forma individual, quanto combinada com outras técnicas estando presente em aproximadamente $60 \%$ das intervenções, conforme apresentado na Tabela 2 .

Tabela 2 - Caracterização da queixa principal e o tratamento aplicado aos colaboradores do Centro Universitário. Teresina, PI $(n=20)$.

\begin{tabular}{lcc}
\hline Queixa Principal & $\mathbf{n}$ & $\mathbf{\%}$ \\
\hline Dor nos pés & 3 & 15,0 \\
\hline Tensão muscular no trapézio & 7 & 35,0 \\
\hline Dor lombar & 5 & 25,0 \\
\hline Dor no ombro & 2 & 10,0 \\
\hline Dor torácica & 3 & 15,0 \\
\hline Intervenção utilizada na pesquisa & 1 & 5,0 \\
\hline Auriculoterapia & 16 & 60,0 \\
\hline Ventosaterapia & 3 & 35,0 \\
\hline Auriculoterapia + Ventosaterapia & 3 & \\
\hline
\end{tabular}

Fonte: Autores (2020).

Hreczuck (2013) realizaram um trabalho com abordagem ergonômica em uma empresa e evidenciou que a principal queixa dos trabalhadores estava relacionada às dores crônicas, principalmente na coluna lombar e cervical, dados que corroboram com o estudo realizado por Global Burden of Disease Study 2013 Collaborators (2015), que apontou que entre 1990 e 2013 a dor lombar foi o sintoma mais citado por pessoas acometidas por dor crônica, e a dor cervical ocupa apenas a quarta entre uma lista de 25 distúrbios que 14 mais acometem a população mundial. Esse achado diverge deste estudo que demostrou que a maioria dos participantes relata tensão muscular no trapézio, porém, dor lombar ocupa a segunda colocação em relação as queixas álgicas.

Campos e Santos (2015) descrevem que a ventosaterapia é uma técnica amplamente utilizada pela fisioterapia para o tratamento de diversas patologias, sejam estes processos dolorosos crônicos ou agudos. A técnica é de fácil aplicação, baixo custo e curta duração, que promove efeitos locais e sistêmicos de forma imediata. O vácuo causado pela sucção promove alívio 
dos processos dolorosos, aumento da circulação sanguínea, relaxamento tecidual, sensação de bem-estar e promove melhora do sono. Fato este que justiça porque a maioria dos participantes deste estudo optaram pela realização deste procedimento, alguns dos pacientes já conheciam as técnicas ofertadas, por divulgação prévia da pesquisa.

A Tabela 3 apresenta a avaliação do nível de dor verificado através da EVA os participantes deste estudo apresentaram média de dor $3( \pm 1,52)$ antes da realização do tratamento, com $80 \%(n=16)$ inferindo dor no escore de 4 a 7 pontos $(\mathrm{p}<0,000)$. Após a intervenção, com uma média de dor de $2( \pm 0,89)$, com $90 \%$ ( $=18$ ) com dor entre 0 a 3 pontos. Quanto a interferência nas atividades laborais apresenta uma média de $6( \pm 1,61)$, com $80 \%$ (n=16) considerando interferência moderada, com redução significativa após a intervenção, apresentando uma média de $2(\llbracket 1,23)$, com 90\% (n=18), relatando interferência de grau leve. Na MTC, o indivíduo é visto como um todo, ele é um ser e deve haver respeito pela escolha. Pois mesmo que o paciente escolha a técnica, se entregarmos o instrumento para o paciente ele não saberá aplicar, os efeitos e tão pouco a duração.

Tabela 3. Caracterização da escala de dor e interferência no trabalho antes e após intervenção. Teresina, PI $(n=20)$.

\begin{tabular}{|c|c|c|c|c|c|c|c|}
\hline Variáveis & & Ante & & & Após & & \\
\hline & Média & $\mathbf{n}$ & $\%$ & $\mathbf{n}$ & $\%$ & Média & Valor de $P$ \\
\hline Escala de dor & $3( \pm 1,52)$ & & & & & $2( \pm 0,89)$ & \\
\hline Entre 0 e 3 & & 0 & 0 & 18 & 90 & & 0,000 \\
\hline Entre 4 e 7 & & 16 & 80 & 2 & 10 & & \\
\hline Entre 7 e 10 & & 4 & 20 & 0 & 0 & & \\
\hline $\begin{array}{l}\text { Interferência da } \\
\text { dor no trabalho }\end{array}$ & $6( \pm 1,61)$ & & & & & $2( \pm 1,23)$ & \\
\hline Leve & & 2 & 10 & 18 & 90 & & 0,019 \\
\hline Moderada & & 16 & 80 & 2 & 10 & & \\
\hline Intensa & & 1 & 10 & 0 & 0 & & \\
\hline
\end{tabular}

Concomitante aos dados apresentados, Garcia, Vieira e Garcia (2013) correlacionam a dor crônica e a atividade laboral, demonstrando que a dor crônica de intensidade moderada está presente na vida de 19\% dos participantes do estudo interferindo de forma moderada a sua atividade profissional. De forma complementar, Moura et al. (2018), identificou redução significativa do escore de intensidade da dor em adultos com dor crônica nas costas, mediante uso da ventosaterapia ( $\mathrm{p}=0.001$ ), comparado com um grupo-controle (cuidado habitual/outra intervenção/lista de espera), concluindo que essa modalidade possui vantagens em aliviar a dor. Dados estes que são concordantes com este estudo e com o estudo de Campos (2015), onde se pode concluir que o uso das ventosas se mostra benéfico em relação à diminuição da dor uma vez que, a técnica proporciona o relaxamento muscular e melhora da circulação sanguínea, facilita a limpeza das glândulas sudoríporas e aumentar a drenagem linfática e circulação venosa, aumento da permeabilidade de membrana, nutrição celular, a mobilização de tecidos subjacentes causa um efeito tonificante e desfibrosante, e ainda há um aumento da liberação de endorfinas no corpo promovendo assim uma resposta analgésica (Agostinho et al., 2006).

A Tabela 4 relaciona a intensidade da dor e seu impacto sobre as atividades de vida diária, social e laboral antes e após as intervenções, apresentando significância para todos os itens avaliados, exceto humor, tendo o item trabalho o melhor avaliado $(0,00( \pm 0,00 ; 0,003 *))$. 
Tabela 4. Valor médio, desvio padrão dos escores do questionário Inventário Breve de Dor (BPI).

\begin{tabular}{|c|c|c|c|}
\hline \multirow[b]{2}{*}{ Variáveis } & \multicolumn{2}{|c|}{ Inventário Breve de Dor (BPI) } & \multirow[b]{2}{*}{ Valor de $\mathrm{p}^{*}$} \\
\hline & Antes & Depois & \\
\hline Pior dor nas últimas 24h & $7,15( \pm 1,66)$ & $3,15( \pm 1,66)$ & 0,000* \\
\hline Melhor dor nas últimas $24 \mathrm{~h}$ & $4,45( \pm 1,85)$ & $1,75( \pm 1,12)$ & $0,014 *$ \\
\hline Média da dor & $5,45( \pm 1,36)$ & $1,90( \pm 1,16)$ & 0,003* \\
\hline Dor agora & $4,95( \pm 1,90)$ & $1,15( \pm 1,31)$ & 0,000* \\
\hline Atividade geral & $5,70( \pm 2,58)$ & $2,15( \pm 1,46)$ & 0,000* \\
\hline Humor & $5,35( \pm 2,50)$ & $0,90( \pm 1,25)$ & $\mathbf{0 , 1 3 2}$ \\
\hline Habilidade de caminhar & $3,50( \pm 1,91)$ & $0,55( \pm 0,76)$ & $0,017 *$ \\
\hline Trabalho & $5,40( \pm 2,76)$ & $0,00( \pm 0,00)$ & 0,003* \\
\hline Sono & $4,10( \pm 2,53)$ & $0,75( \pm 0,64)$ & 0,075* \\
\hline Habilidade de apreciar a vida & $2,00( \pm 2,86)$ & $0,10( \pm 0,48)$ & 0,008* \\
\hline
\end{tabular}

Fonte: Autores (2020).

De acordo com o estudo de Bastos (2015), a MTC deve ser parte integrante do conhecimento dos profissionais da saúde. Segundo o autor a utilização das técnicas que contemplam a MTC atuam de forma integrante e complementar ao tratamento convencional, proporcionando o alívio dos sintomas e minimizando o uso de fármacos (Rosa, Dias \& Roncada 2016).

Tolentino (2016) aplicou a técnica de auriculoterapia em adultos com dor lombar crônica, mostrando ter eficácia quanto a dor, funcionalidade e mobilidade. Estudo semelhante realizado por Silvério (2013) demonstra que as técnicas da MTC são eficazes na redução da dor, diminuição na utilização de fármacos e na melhora da qualidade de vida. Os dados descritos na literatura concernem com os obtidos neste estudo, sendo melhor evidenciados na Tabela 5, na qual apresenta a significância entre as variáveis dor $\left(2( \pm 0,89) ; 0,000^{*}\right)$, interferência no trabalho $\left(2( \pm 1,23) ; 0,000^{*}\right)$ e média da dor $\left(1,90( \pm 1,16) ; 0,003^{*}\right)$.

Tabela 5. Comparativo estatístico das variáveis analisadas. Teresina, PI $(n=20)$.

\begin{tabular}{lccc}
\hline Características & Antes & Depois & Valor de p* \\
\hline Escala de dor & $3( \pm 1,52)$ & $2( \pm 0,89)$ & $\mathbf{0 , 0 0 0} *$ \\
\hline Interferência da dor no trabalho & $6( \pm 1,61)$ & $2( \pm 1,23)$ & $\mathbf{0 , 0 0 0} *$ \\
\hline Média da dor & $5,45( \pm 1,36)$ & $1,90( \pm 1,16)$ & $\mathbf{0 , 0 0 3} *$ \\
\hline
\end{tabular}

Comparativo das médias de escalas de dor, interferência da dor no trabalho e qualidade de vida antes e média da dor no questionário Inventário Breve de Dor (BPI) test t-student para amostras pareadas. Fonte: Autores (2020).

Este estudo demonstra a intensa necessidade de suporte junto às atividades laborais, enfatizando a importância de assistência aos colaboradores como meio de promover qualidade de vida no ambiente de trabalho e com isso, melhor desempenho em suas atividades. O estudo apresenta como limitação a quantidade de profissionais participantes, uma vez que, os atendimentos ocorriam nos intervalos das atividades, nem todos podendo se fazer presentes. Outro ponto importante, visando a perspectiva de mais estudos dentro desta temática, buscar investigar qual a técnica de MTC mais favorável na reversibilidade do quadro sintomático, comparando estes de forma isolada ou combinada, com intuito de melhor caracterizar o atendimento à população atendida.

\section{Conclusão}

No estudo houve predominância do gênero feminino, porém dentre os colaboradores participantes não houve um padrão quanto aos cargos atendidos, o que vem diversificar a queixa principal dos mesmos, contudo, com maior predominância 
para as áreas do trapézio e da coluna lombar, sendo estas aferidas dores de grau intermediária a grave, com moderado impacto nas atividades laborais, no entanto, a aplicação das técnicas de MTC apresentaram resultado satisfatório em todas as variáveis, com ênfase no desempenho do trabalho, o que implica na eficácia das técnicas e na modalidade de atendimento, abrindo viés para aplicabilidade destas técnicas nos ambientes de trabalho, uma vez que são avaliadas como segura, simples e de baixo custo.

\section{Referências}

Agostinho, A, Cordeiro, A. Leiria, D. Brandão, D. Tiago, J. \& Pedro, L. (2016). Vacuoterapia: influência no aumento da flexibilidade muscular dos isquiotibiais. Saúde \& Tecnologia, 16: 38-43.

Alencar, M. C. B., \& Merlo A. R. C (2018). A saúde em troca da excelência: o sofrimento de atendentes de nutrição de um hospital público acometidos por LER/Dort. Saúde soc. 27(1): 2015- 226.

Bastos, R. A (2015). A sintomatologia do stress sob a ótica da medicina tradicional chinesa. ABCS Health Science, 40(2): 96-101.

Campos, G. H., \& Santos, C. T (2015). Tratamento de pontos-gatilhos (trigger points) por meio de terapia por ventosa. Revista de Divulgação Científica Sena Aires, 4(2): 146-154.

Cintra, M. E. R (2012). Percepções de Corpo Identificadas entre Pacientes e Profissionais de Medicina Tradicional Chinesa do Centro de Saúde Escola do Butantã, Saúde Soc. v.21, n.1, p.193-205.

Garcia, B. T., Vieira, E. B. M., \& Garcia, J. B. S (2013). Relação entre dor crônica e atividade laboral em pacientes portadores de síndromes dolorosas. Revista dor, v. 14, n. 3, p. 204- 209, set.

Global Burden Of Disease Study 2013 Collaborators. Global (2015) regional, and national incidence, prevalence, and years lived with disability for 301 acute and chronic diseases and injuries in 188 countries, 1990-2013: a systematic analysis for the Global Burden of Disease Study 2013. Lancet. 386(9995):743800. doi: 10.1016/S0140-6736(15)60692-4..

Hreczuck, D. V., Ulbricht, L. (2013). Prescrição de um programa de ginástica laboral para o trabalho frente ao computador: uma abordagem econômica. Revista Uniandrade, 12, 112-124.

Kim, D. S. Suma de Diagnósticos Secretos para Tratamento com Ventosa-Terapia. Dong Yang, 2002,

Lu, A. P., Jia, H. W., Xiao, C., \& Lu, Q. P. (2004). Theory of traditional Chinese medicine and therapeutic method of diseases. World J Gastroenterol [Internet],10(13):1854-6,

Martinez, J. E, Grassi, D. C, \& Marques, L. G (2011). Análise da aplicabilidade de três instrumentos de avaliação de dor em distintas unidades de atendimento: ambulatório, enfermaria e urgência. Rev. Bras. Reumatol., 51, 304-308, <http://www.scielo.br/scielo.php?script=sci_arttext\&pid=S048250042011000400002\&lng=en\&nrm=iso>. http://dx.doi.org/10.1590/S0482-50042011000400002.

Matos E, \& Pires D (2006). Teorias administrativas e organização do trabalho: de Taylor aos dias atuais, influências no setor saúde e na enfermagem. Texto contexto enferm, 15(3):508-514,

Ministério da saúde. Secretaria de Atenção à Saúde, Secretaria de Gestão do Trabalho e da Educação na Saúde. Guia prático do Cuidador. Brasília (DF): MS: 2008. http://bvsms.saúde.gov.br/publicacoes/guia_guiapratico_cuidador.pdf_

Moreno, A. G. U. T, Bezerra, A. G. V, Silva, E. G. A, Melo, E. L, Gerbi, M. E. M. M, Bispo, M. E. A, SÁ, R. A. G, \& Menezes, M. R. A (2021). Influência do estrógeno na modulação da dor na disfunção temporomandibular e sua prevalência no sexo feminino: revisão integrativa. Research, Society and Development, v. 10, n. 2, e38510212453. http://dx.doi.org/10.33448/rsd-v10i2.12453

Moura, C. C., Chaves, C. L., Cardoso, A. C. L. R., Nogueira, D. A., Corrêa, H. P., \& Chianca, T. C. M. (2018) Ventosaterapia e dor crônica nas costas: revisão sistemática e metanálise. Rev. Latino-Am. Enfermagem [Internet]. 26: e3094. https://doi.org/10.1590/1518-8345.2888.3094.

Moura, C. C, \& Chianca, M. C. T. Efeitos da associação da ventosaterapia à acupuntura auricular sobre a dor crônica nas costas: ensaio clínico randomizado. Repositório UFMG, 2018 https://repositorio.ufmg.br/handle/1843/31108

Nascimento, M. C. do. As duas faces da montanha: estudos sobre medicina chinesa e acupuntura. Hucitec, 2006.

Organização Mundial Do Trabalho, Organização Pan-Americana De Saúde. Estresse no ambiente de trabalho cobra preço alto de indivíduos, empregadores e sociedade. http://www.paho.org/bra/index.php?option=com_content\&view=article\&id=5087\%3Aestresse- no-ambiente-de-trabalho-cobra-preco-alto-deindividuos-empregadores-e-sociedade \&Itemid=839. Acesso em 04/05/2017,

Pandolphi, Costa, I. C. C. (2016) Vista da análise das ler/dort nofiticadas no estado do rio grande do Norte de 2010 a 2014 . Revista ciência plural. 2(3):82-96

Rosa, R., Dias, C. P., \& Roncada, C. (2016). Efeitos da acupuntura na redução da dor lombar: uma revisão da literatura. Revista Pesquisa em Fisioterapia. 6(2):167-178.

Rodrigues, K. A., Pachêco, J. M. S., Nascimento-Júnior, C. P., Alves, G. B., Carvalho, R. M. A., DantasNeta, N. B., Cantinho, K. C. R., \& Carvalho, G. D. (2021). Impacto do calçado com salto em mulheres de 18 a 35 anos. Brazilian Journal of Development. 7(4): 41001-41014. 
Research, Society and Development, v. 10, n. 10, e33101017951, 2021

(CC BY 4.0) | ISSN 2525-3409 | DOI: http://dx.doi.org/10.33448/rsd-v10i10.17951

Silva, E. L. (2012). Auriculoterapia como ferramenta de cuidado em saúde na atenção básica: relato de experiência.universidade de pernambuco- upe faculdade de ciências médicas. Editora realize. http://editorarealize.com.br/revistas/congrepics/trabalhos/TRABALHO_EV0 76_MD4_SA1_ID357_27082017154740.pdf.

Silva, E. A. C, \& Gomes, A. F. R. (2016). Análise da relação entre uso de calçados de salto alto e desenvolvimento de disfunções biomecânicas: uma revisão bibliográfica. Editora realize.

Silvério, L. S., \& Seroiska, M. A. (2013). Acupuntura para analgesia. In: Silvério-Lopes, S. (Ed.), Analgesia por Acupuntura. Curitiba, Brasil: Omnipax, p. 122.

Tolentino, F. (2016). Efeito de um tratamento com auriculoterapia na dor, funcionalidade e mobilidade de adultos com dor lombar crônica. Dissertação. Universidade Estadual Paulista, Instituto de Biociências de Rio Claro.

Uva, A. S. (2009). Salud y Seguridad del Trabajo en Portugal: apuntes diversos. Med Segur Trab. 55(214):12-25,

Viegas, L. R. T., \& Almeida, M. M. C. (2016). Perfil epidemiológico dos casos de LER/DORT entre trabalhadores da indústria no Brasil no período de 2007 a 2013. Rev. bras. saúde ocup. 41:e22. http://dx.doi.org/10.1590/2317-6369000130615.

World Health Organization. (2006). Constitution of the World Health Organization. Basic Documents [on line]. http://www.who.int/governance/eb/who_constitution_en.pdf

Yamamura, Y. (2006). Entendendo medicina chinesa e acupuntura. Centre AO. 